\title{
Clinical Investigation: Determinants of Blood Cell Omega-3 Fatty Acid Content
}

\author{
Robert C. Block*,a, William S. Harris ${ }^{\mathrm{b}}$ and James V. Pottala ${ }^{\mathrm{b}}$ \\ ${ }^{a}$ Division of Epidemiology, Department of Community and Preventive Medicine, University of Rochester School of \\ Medicine and Dentistry, USA \\ ${ }^{b}$ Nutrition and Metabolic Disease Research Center, Sanford Research/USD, Sioux Falls, South Dakota, USA
}

\begin{abstract}
Background: Although red blood cell eicosapentaenoic acid (EPA) plus docosahexaenoic acid (DHA) content (the Omega-3 Index) predicts cardiovascular death, the factors determining the Index are unknown.

Methods: In 704 outpatients, we undertook an investigation of the clinical determinants of the Index.

Results: Factors associated with the Index in decreasing order were: EPA+DHA supplement use, fish consumption frequency, triglyceride level, age, high cholesterol history, and smoking. These factors explained 59\% of Index variability, with capsules/fish intake together accounting for $47 \%$. The Index increased by $13 \%(p<0.0001)$ for each serving level increase in fish intake and EPA+DHA supplementation correlated with a 58\% increase $(p<0.0001)$ regardless of background fish intake ( $p=0.25$; test for interaction). A $100 \mathrm{mg} / \mathrm{dL}$ decrease in serum triglycerides was associated with a $15 \%$ higher $(p<0.0001)$ Index.

Conclusions: The intake of EPA+DHA-rich foods and supplements principally determined the Omega-3 Index, but explained only about half of the variability.
\end{abstract}

\section{INTRODUCTION}

Increased content of the omega- 3 fatty acids eicosapentaenoic acid (EPA) and docosapentaenoic acid (DHA) in whole blood [1], plasma phospholipids [2] or red blood cells [3] has been associated with a markedly reduced risk for sudden cardiac death. We recently proposed that the EPA and DHA content of red blood cell membranes, expressed as a percent of total membrane fatty acids (i.e., the Omega-3 Index), be considered as a possible new cardiovascular disease risk factor, particularly for sudden cardiac death [3]. Increased membrane EPA and DHA content are known to modulate the activity of a variety of membrane-associated proteins including ion channels and interleukin-1 receptors [4]. Because the presence of these long-chain omega- 3 fatty acids in membranes may directly influence cell function, notably myocardial electrical stability $[4,5]$, it is reasonable to consider that some measure of membrane omega-3 content (especially one that is highly correlated with that of human cardiac tissue [6]) may serve as a risk marker. Indeed, we recently reported that a reduced omega- 3 blood level was associated with increased odds for acute coronary syndromes in young [7] and more elderly [8] individuals.

If blood cell omega- 3 content is to be used as a risk factor, then the factors that influence this marker need to be elucidated. In a small series of healthy volunteers, we found that the most important determinant of the Omega-3 Index

*Address correspondence to this author at the Division of Epidemiology, Department of Community and Preventive Medicine, University of Rochester School of Medicine and Dentistry, Box 644, 601 Elmwood Avenue, Rochester, New York 14642, USA; Tel: (585) 233-7265; Fax: (585) 4614532; E-mail: robert_block@urmc.rochester.edu was, not surprisingly, the intake of oily fish (tuna/other nonfried fish) [3]. But unexpectedly, age and BMI were also predictors independent of fish intake. Hence, there is a need for a more focused examination of the clinical, anthropometric, and lifestyle factors that, together, influence the Omega3 Index.

\section{METHODS}

\section{Subjects}

Seven hundred sixty-eight subjects were recruited as controls between March 8, 2004 and March 1, 2005 as part of a case-control study examining the relationship between blood fatty acid composition and the presence of an acute coronary syndrome. The relationship between acute coronary syndrome status and blood cell EPA and DHA has been previously reported for this case-control study cohort [8]. Permission was sought from male and non-pregnant female outpatients age 35 and older having blood drawn for routine clinical testing at blood drawing centers at Saint Luke's Hospital in Kansas City. By selecting patients from an outpatient clinical laboratory, we were able to include subjects with medical issues warranting care. A history of coronary heart disease or other chronic diseases did not exclude them from participation. One additional 10-cc EDTA tube was obtained from each subject, and each was asked to complete a 2-page questionnaire to capture demographic and relevant medical history information. This 2-page questionnaire included questions regarding demographics, personal medical and family history, medications, fish and fish oil intake. Fish intake was categorized as: never, less than 1 serving per month, 1-3 servings per month, 1 serving per week, at least 2 servings per week, as the answer to this question has been 
shown to be a powerful predictor of red blood cell EPA+DHA content [3,9] and risk for death from ischemic heart disease in the Cardiovascular Health Study [10]. The study was approved by the hospital's institutional review board and conformed to the guidelines outlined in the Declaration of Helsinki.

\section{Laboratory Methods}

Blood cell fatty acid composition of EPA and DHA was measured as previously described [8]. Briefly, cell membranes were isolated by ultracentrifugation, and membrane fatty acids were converted to methyl esters by heating them at $100^{\circ} \mathrm{C}$ for $10 \mathrm{~min}$ in $14 \%$ boron trifluoride. Extracted methyl esters were analyzed by gas chromatography. A custom-made mixture of fatty acids (designed to mimic erythrocyte fatty acid composition; GLC 673b, Nuchek Prep, Elysian, MN) was included as an external standard with each run for peak identification and for response factor adjustment. The response factor for palmitic acid was assumed to be 1.0, and that for EPA and DHA was calculated based on this assumption. Fatty acid peak areas were adjusted on a daily basis using these response factors and reported as a percent of the total area for identified peaks. The coefficient of variation for EPA+DHA was 5\%. Serum lipids were measured in the hospital clinical laboratory by routine enzymatic methods. In those samples with triglycerides under $400 \mathrm{mg} / \mathrm{dL}$, low density lipoprotein cholesterol (LDL-C) was calculated by the Friedewald equation [11]; otherwise LDL$\mathrm{C}$ was not reported.

\section{Statistical Analysis Method}

A multivariable log-normal regression model was developed to determine variables associated with the marker of primary interest, i.e., the percent EPA+DHA in blood cell membranes (Omega-3 Index). Mallow's CP method of model selection [12] was used to identify parsimonious models. Additional covariates selected a priori to be in the model were: BMI, gender, HDL-C, LDL-C, and diabetes mellitus (yes/no). A separate model with the same final predictor variables was fitted to the 228 women in the study of which 217 could be classified as either estrogen sufficient (pre-menopausal or post-menopausal taking hormone replacement therapy) or estrogen deficient (post-menopausal not taking hormones). Separate models with the same predictor variables were also fitted to those individuals with and those individuals without a diagnosis of prior myocardial infarction, or revascularization via coronary artery bypass graft surgery or percutaneous transluminal coronary angioplasty. Diagnostics were conducted to verify model assumptions. A $p$-value $<0.05$ was used to ascribe statistical significance. SAS ${ }^{\circledR}$ version 9.1 (Cary, N.C) software was used for all analyses. The authors had full access to the data and take responsibility for its integrity. All authors have read and agree to the manuscript as written.

\section{RESULTS}

Blood samples and questionnaires were available from all 768 subjects. Since the cohort was overwhelmingly Caucasian (91.7\%) [African American (7.9\%), other (0.4\%)] the analyses were restricted to this study group as it was the only racial category large enough to consider the interaction effect between fish oil supplements and fish intake. These subjects $(n=704)$ were $67 \%$ male with a BMI of $28 \pm 6$ and an age of $62 \pm 12$ years. Fourteen percent of individuals had a diagnosis of a prior myocardial infarction or revascularization. Fish oil supplements were being taken by $34 \%, 13 \%$ were smokers, and $16 \%$ of subjects were reported as having diabetes mellitus (Table 1). The average Omega-3 Index (EPA+DHA content of blood cells) for the study group was $4.3 \% \pm 2.0 \%$ (mean $\pm \mathrm{SD}$ ). For those individuals who did not report taking fish oil supplements it was $3.4 \% \pm 1.3 \%$ and for those on supplements it was $6.0 \% \pm 2.1 \%(\mathrm{p}<0.0001)$.

Table 1. Description of Subjects

\begin{tabular}{|c|c|c|}
\hline Variable & $\mathbf{N}$ & \\
\hline Age & 704 & $62 \pm 12^{\dagger}$ \\
\hline BMI & 666 & $28 \pm 5.7$ \\
\hline Weight (lbs) & 700 & $187 \pm 43$ \\
\hline Triglycerides (mg/dL) & 704 & $167 \pm 132$ \\
\hline $\operatorname{HDL}(\mathrm{mg} / \mathrm{dL})$ & 704 & $51 \pm 16$ \\
\hline LDL (mg/dL) & 679 & $108 \pm 38$ \\
\hline Cholesterol (mg/dL) & 704 & $191 \pm 45$ \\
\hline Male & 701 & $473(67)$ \\
\hline Diabetes mellitus & 652 & $104(16)$ \\
\hline Currently smoking & 687 & $92(13)$ \\
\hline Fish Intake & 693 & \\
\hline Never & & $55(7.9)$ \\
\hline Less than $1 /$ month & & $194(28)$ \\
\hline $1-3$ per month & & $233(33.6)$ \\
\hline $1 /$ week & & $137(19.8)$ \\
\hline At least $2 /$ week & & $74(10.7)$ \\
\hline Fish oil supplements & 696 & $235(34)$ \\
\hline High cholesterol (by history) & 674 & $387(57)$ \\
\hline Some college vs. none & 696 & 547 (79) \\
\hline Myocardial infarction (by history) & 692 & $92(13)$ \\
\hline Premature CHD (by history) & 701 & $144(21)$ \\
\hline Family history of premature CHD & 691 & $253(37)$ \\
\hline Hypertension (by history) & 679 & $368(54)$ \\
\hline Some alcohol use vs. none & 704 & $506(72)$ \\
\hline Estrogen sufficient & 217 & $81(37)$ \\
\hline
\end{tabular}

${ }^{\dagger}$ Mean $\pm \mathrm{SD}$; Values in parentheses are percent.

Seven parsimonious models were identified which reduced the original 20 covariates (Table 1) to the following 8 that were significant covariates in all seven models: supplementation with fish oil capsules (yes/no), fish intake, age, triglycerides, current smoker (yes/no), history of myocardial infarction (yes/no), hypertension (yes/no), and high choles- 
terol (yes/no). The inclusion of additional covariates (BMI, gender, HDL, LDL, and diabetes mellitus) resulted in two model components (history of myocardial infarction and hypertension) becoming insignificant $(p>0.1)$ and were therefore dropped from the final model (Fig. 1). A total of 551 subjects had complete data for these 11 variables, and therefore only these subjects were included in the final model. In multivariable regression analysis, the primary determinants of the Omega-3 Index were the frequency of nonfried fish intake and the use of fish oil supplements (Fig. 1), however, there was no interaction between the two $(p=$ 0.25 ). Modeling fish intake as a continuous variable fit the data as well as modeling categorically by the likelihood ratio test $\left(X^{2}(3)=1.59, p=0.66\right)$. The Omega-3 Index was $13.2 \%$ higher for each increasing category of fish intake, and the effect of supplementation multiplied the Omega-3 Index by $58.1 \%$ regardless of the background fish intake (Fig. 2).

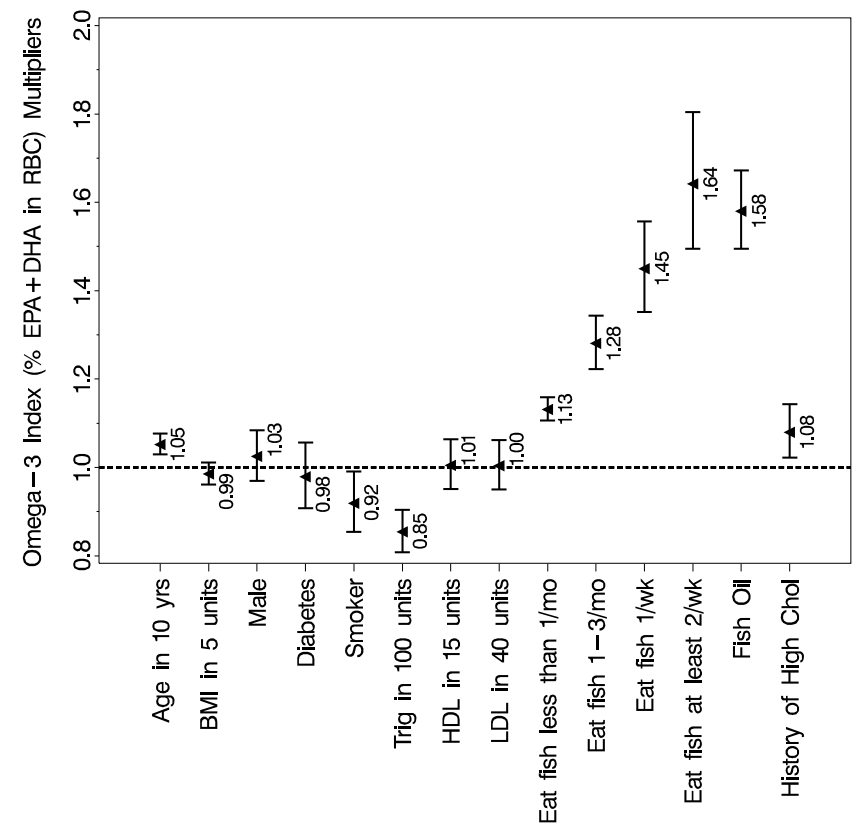

Fig. (1). Multiplier effects on the Omega-3 Index for all predictors included in the final multivariable log-normal regression model. The fish consumption estimates are compared to the reference of never eating fish. Data are presented as point estimates with $95 \%$ confidence intervals.

Other significant independent determinants positively associated with the Omega-3 Index were age $(+5.3 \%$ for a 10 year increase), and a history of high cholesterol $(+8.1 \%)$. Negatively associated factors were being a current smoker ($8.0 \%)$ and triglycerides $(-14.5 \%$ per $100 \mathrm{mg} / \mathrm{dL})$. Factors that were not significant predictors of the Omega-3 Index included: gender, diabetes mellitus, BMI, weight, HDL, LDL, total cholesterol, some college education vs. none, some alcohol use vs. none, and a self-reported history of myocardial infarction, coronary heart disease, hypertension, or a family history of coronary heart disease. Estrogen status was not associated with the Omega-3 Index $(p=0.09)$. Being a smoker was also not a significant predictor of the Omega-3 Index ( $p$-value $=0.53$ ) for women when adjusting for estrogen status. When we adjusted for coronary heart disease status, the results did not change (data not shown).

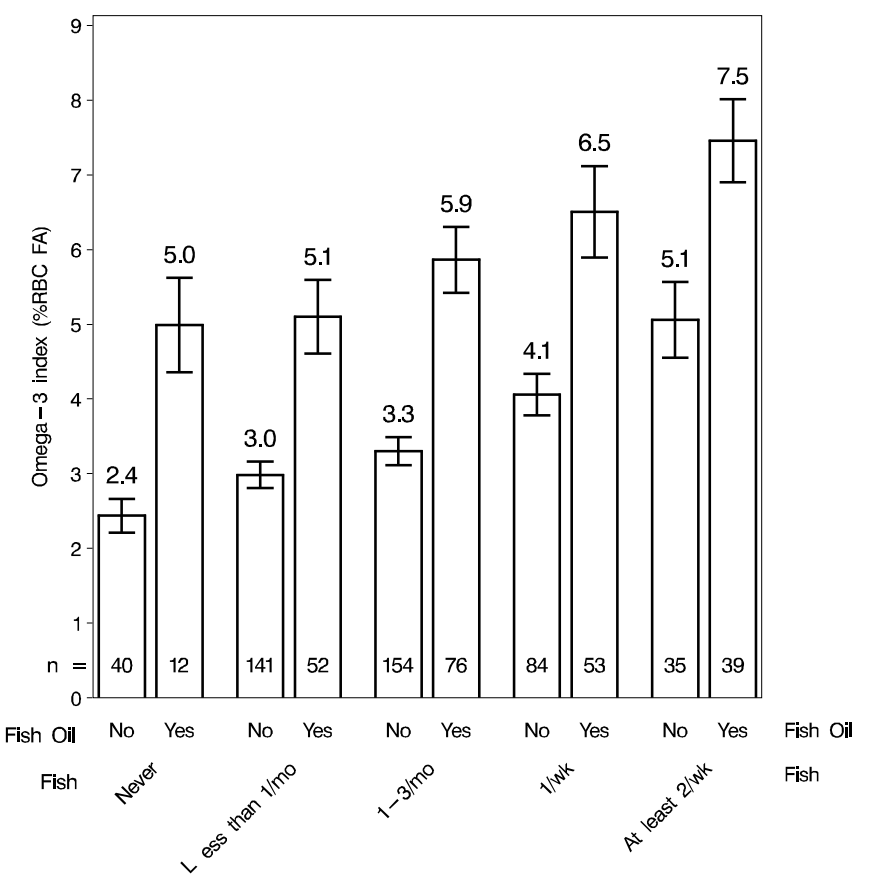

Fig. (2). Raw data of the Omega-3 Index with $95 \%$ confidence intervals for each group with a combination of fish intake frequency and fish oil supplementation status, including group samples sizes.

The final model (Fig. 1) explained $59 \%$ of the variation in the Omega-3 Index. Fish oil supplementation and fish intake together accounted for $47 \%$ of the variation, with the former explaining somewhat more of the variance than the latter. The multivariable linear regression equation generated is as follows:

Eq. (1) Score $=0.77+0.05 *($ Age $/ 10)+0.46$ (if taking fish oil) + [choose only 1 category: 0.000 (if never eating fish) +0.12 (if eating fish $<1 / \mathrm{mo}$ ) +0.25 (if eating fish $1-3 / \mathrm{mo}$ ) +0.37 (if eating fish $1 / \mathrm{wk}$ ) + 0.50 (if eating fish $\geq 2 / \mathrm{wk}$ ) +0.08 (if history of high cholesterol $)-0.16^{*}($ Triglycerides $/ 100)-0.08$ (if current smoker).

Eq. (2) Omega-3 Index $=\operatorname{Exp}($ Score $)$

\section{DISCUSSION}

This study was undertaken to begin to define the factors (demographic, clinical, lifestyle, dietary) that determine the content of EPA+DHA in blood cell membranes (the Omega3 Index). In this sample of 704 outpatients drawn from a large metropolitan hospital in the Midwest, the average Omega-3 Index for those individuals who did not report taking fish oil supplements was $3.4 \%$, and for those taking supplements it was $6.0 \%$. The former is lower than the $4.9 \%$ reported in healthy volunteers [3], and below the high-risk level of $4 \%$ or less that has recently been proposed [3]. The $6.0 \%$ score, although significantly higher, is below the proposed $8 \%$ or higher target value associated with significantly reduced risk for coronary heart disease death [3]. The intake of omega-3 fatty acids required to achieve "cardioprotective" blood levels has not been established, but intakes of 500 to $1,000 \mathrm{mg} /$ day of EPA + DHA have been suggested [3] and 
are consistent with current American Heart Association guidelines [13]. This level of intake can typically be achieved by consuming 2 to 3 servings per week of oily fish such as albacore tuna, mackerel, herring, salmon, or sardines, or by taking 2-3 standard over-the-counter fish oil capsules each day. However, in this study individuals consuming that amount of fish and taking a supplement still did not achieve levels demonstrated to be most protective [3]. Even in those who reported eating fish at least twice each week and also taking an omega-3 supplement, the average level of $7.5 \%$ found is less than this target of $8 \%$. Thus, although supplementation was associated with higher levels, even greater intake than that in this highest intake group appears necessary to achieve levels associated the greatest reduction in risk for sudden cardiac death. Of note, red blood cell EPA+DHA varied over 7-fold in this sample. Complicating these public health implications of low intake of EPA+DHA is that, while we assumed that much of the variance was due to differences in EPA+DHA intake, the extent to which intake determines the Omega-3 Index is not clear.

The number of servings of oily fish consumed per month did indeed have a major effect on long-chain omega-3 fatty acid blood levels (Fig. 2). This was, of course, expected and suggests that simply inquiring about the number of tuna or non-fried fish meals that a patient is consuming on a monthly basis will provide a reasonable estimate of his/her red blood cell EPA+DHA. This finding confirms what has already been shown with omega-3 fatty acid capsules; i.e., that the EPA+DHA content of red blood cell membranes provides a good estimation of omega-3 fatty acid intake [3].

As observed in a previous cohort [9] of only 163 subjects, increasing age was an independent predictor of higher red blood cell EPA+DHA, even after adjusting for fish and fish oil capsule intake. This is a robust association as it is also consistent with findings in very different populations: the Inuit of Nunavik, the James Bay Cree, and residents of metropolitan Quebec City [14-16]. Since detailed information about omega-3 fatty acid intake was not obtained in this study, it is possible that older individuals taking supplements might take more capsules, use more highly-concentrated capsules, or take them more consistently than younger persons who supplemented. There may, however, be a nondietary basis for this observation since older rats have higher cardiac DHA levels than younger rats fed the same diet [17]. One could speculate that older individuals may consume more alpha-linolenic acid or may convert it to longer chain omega- 3 fatty acids more readily than younger people. Finally, the correlation between higher a Omega-3 Index and age could reflect an increasingly slower turnover of omega-3 fatty acids in tissues. More studies in humans are needed to explore these possibilities given that current recommendations for omega-3 fatty acid intake for individuals with and without coronary heart disease do not differ by age [13].

An increasing body mass index was not associated with the Omega-3 Index independent of fish and supplement intake. A simplistic interpretation of this observation may be that the volume of distribution of EPA+DHA in blood does not vary significantly in the setting of variations in the distribution of adipose tissue. These findings contrast with those of Cazzola et al. [18] who found a lower omega-3 fatty acid content of red blood cell membranes in overweight and obese subjects than those of normal weight. These authors found that red blood cells from these individuals were more susceptible to oxidative stress, suggesting that oxidation may be a mechanism for reducing omega-3 levels. The difference between the findings of these two studies may be due to the fact that Cazzola, et al. classified individuals as either normal weight (BMI <25) or overweight-obese (BMI 25-33) and the majority of our subjects were overweight or obese but classified as obese only if their body mass index was $>30$.

We did not find a gender-related difference in the Omega-3 Index, confirming our previous observation [9] in a much smaller cohort of 163 subjects. It has been recently reported that women may have a greater capacity to synthesize long-chain from short-chain omega-3 fatty acids [19, 20], and tend to have higher plasma DHA levels than men $[19,21]$. This may reflect their physiological need to provide pre-formed DHA to the developing fetus. Hence, we expected that the women in our survey would have higher proportions of EPA+DHA in blood cell membranes than the men. We, however, found no relationship between gender and the Omega-3 Index, and no interaction between gender and supplementation status. This indicates that the lack of an association of gender with the Omega-3 Index was seen in those women taking fish oil supplements and those not doing so. Hence gender alone (at least among older subjects) may not be an important determinant of blood cell EPA+DHA.

Although in our previous study [9] we found no relation between the Omega-3 Index and smoking status, in the present study smokers had, on average, an $8 \%$ lower Index than non-smokers. This is in agreement with some [7, 22-25], but not other observations $[26,27]$. The contrast with the findings in our prior study may be due to that study's much smaller sample size (163) or to the fact that the current study was limited to Caucasians whereas the prior study included $18 \%$ non-Caucasians. The pro-oxidative state characteristic of smokers [28] might be expected to accelerate the destruction of long chain polyunsaturated fatty acids like EPA and DHA. Another observation that failed to confirm findings from our previous study related to diabetes mellitus. Here we found that the presence of diabetes mellitus was not associated with the Omega-3 Index whereas in our earlier study these were inversely related [9]. A reduced Omega-3 Index in diabetic subjects has been reported by some [29] but not other investigators [30-32]. A reduced EPA level might be expected in individuals with diabetes because the delta- 6 and delta-5 desaturases (enzymes responsible for converting $\alpha$ linolenic acid to long chain n-3 FA) have been reported to be inhibited $[33,34]$.

The evidence for a lower risk of ischemic cardiac death in those with higher levels of EPA and DHA is strong (see review by Wang, et al. [35]). Recent evidence also has shown that, in the setting of the typical United States diet, low whole blood levels of EPA and DHA are an independent risk factor for other acute coronary syndromes [7]. Although supplemental EPA and DHA is known to reduce serum triglyceride levels (high levels are a cardiovascular disease risk factor [36]), the data from this adult cohort along with that from a middle-aged segment of the same cohort [7], are some of the first to demonstrate that triglyceride levels are associated with blood Omega-3 Index levels. These findings 
suggest that part of the reduced risk for coronary heart disease in subjects with higher blood levels of EPA and DHA may be mediated by changes in blood lipids, in contrast to prior studies that have suggested that risk reduction is independent of triglyceride levels $[1,37,38]$.

\section{LIMITATIONS AND FUTURE DIRECTIONS}

A limitation of this study is that it is cross-sectional in nature, temporal relationships cannot be established, and thus causal inferences cannot be made. This study was limited to Caucasians and thus generalizability to other populations is limited. Although we were able to record the frequency of fish intake, fish oil intake information was limited to whether or not an individual was or was not taking a supplement. Thus, our estimate of the contribution of supplementary EPA and DHA to the Omega-3 Index is likely to be rough. Although we did not investigate the contribution of dietary alpha linolenic acid [(ALA); a plant-derived omega-3 fatty acid] to the Omega-3 Index, the focus of the study was on the fish-derived omega-3 fatty acids which compose the Index. Finally, this was a single center study. Strengths of this study included the relatively large sample size, the use of a validated and very simple fish intake questionnaire, and the analysis of blood cell membrane fatty acid levels which has been validated against human myocardial tissue EPA and DHA levels [6].

Further studies are warranted to more carefully define the factors, both omega-3 fatty acid intakes and other demographic and lifestyle-related, that impact this emerging coronary heart disease risk factor. In addition, studies should focus specifically on individuals at increased risk for sudden cardiac death to determine if the determinants of the Omega3 Index vary from controls in this at-risk population. In addition, the impact of differing ratios of dietary EPA and DHA as well as alpha-linolenic acid and other dietary fatty acids on this biomarker will need to be examined.

\section{ACKNOWLEDGEMENTS}

The authors wish to express their appreciation to Scott Sands, Mary Baston and Lan Zhang for their important contributions in sample processing. We are deeply indebted to Dr. James Crockett and the Saint Luke's Hospital Foundation for providing funding for this study and for Donna Vink in manuscript preparation.

\section{FINANCIAL SUPPORT}

This project was funded by a grant from the Saint Luke's Hospital Foundation, Kansas City, MO, and Grant Number KL2 RR 024136 from the National Center for Research Resources (NCRR), a component of the National Institutes of Health (NIH), and the NIH Roadmap for Medical Research. Its contents are solely the responsibility of the authors and do not necessarily represent the official view of NCRR or NIH. Information on NCRR is available at http://www.ncrr.nih. gov/. Information on Re-engineering the Clinical Research Enterprise can be obtained from http://nihroadmap.nih.gov/ clinicalresearch/overview-translational.asp.

\section{ABBREVIATIONS}

$\mathrm{EPA}=$ Eiocosapentaenoic acid

DHA $=$ Docosahexaenoic acid
$\mathrm{BMI}=$ Body mass index

LDL = Low density lipoprotein

HDL = High density lipoprotein

\section{REFERENCES}

[1] Albert CM, Campos H, Stampfer MJ, et al. Blood levels of longchain $\mathrm{n}-3$ fatty acids and the risk of sudden death. $\mathrm{N}$ Engl J Med 2002; 346: 1113-8.

[2] Lemaitre RN, King IB, Mozaffarian D, Kuller LH, Tracy RP, Siscovick DS. n-3 polyunsaturated fatty acids, fatal ischemic heart disease, and nonfatal myocardial infarction in older adults: The Cardiovascular Health Study. Am J Clin Nutr 2003; 77: 319-25.

[3] Harris WS, von Schacky C. The Omega-3 Index: a new risk factor for death from coronary heart disease? Prev Med 2004; 39: 212-20.

[4] Leaf A, Kang JX, Xiao Y, Billman GE. Clinical prevention of sudden death by n-3 polyunsaturated fatty acids and mechanism of prevention of arrhythmias by $n-3$ fish oils. Circulation 2003; 107: 2646-52.

[5] Kang JX, Leaf A. Prevention of fatal cardiac arrhythmias by polyunsaturated fatty acids. Am J Clin Nutr 2000; 71: 202S-7S

[6] Harris WS, Sands SA, Windsor SL, et al. Omega-3 fatty acids in cardiac biopsies from heart transplantation patients: correlation with erythrocytes and response to supplementation. Circulation 2004; 110: 1645-9.

[7] Harris WS, Reid KJ, Sands SA, Spertus JA. Blood omega-3 and trans fatty acids in middle-aged acute coronary syndrome patients Am J Cardiol 2007; 99: 154-8.

[8] Block RC, Harris WS, Reid KJ, Sands SA, Spertus JA. EPA and DHA in blood cell membranes from acute coronary syndrome patients and controls. Atherosclerosis 2008; 197(2): 821-8.

[9] Sands SA, Reid KJ, Windsor SL, Harris WS. The impact of age, body mass index, and fish intake on the EPA and DHA content of human erythrocytes. Lipids 2005; 40: 343-7.

[10] Mozaffarian D, Lemaitre RN, Kuller LS, et al. Cardiac benefits of fish consumption may depend on the type of fish meal consumed: the Cardiovascular Health Study. Circulation 2003: 107: 1372-7.

[11] Friedewald WT, Levy RI, Fredrickson DS. Estimation of the concentration of low-density lipoprotein cholesterol in plasma, without use of the preparative ultracentrifuge. Clin Chem 1972; 18: 499502.

[12] Mallows CL. Some comments on Cp. Technometrics 1973; 15: 661-75.

[13] Kris-Etherton PM, Harris WS, Appel LJ. Fish consumption, fish oil, omega-3 fatty acids, and cardiovascular disease. Circulation 2002; 106: 2747-57.

[14] Dewailly E, Blanchet C, Lemieux, S. et al. n-3 Fatty acids and cardiovascular disease risk factors among the Inuit of Nunavik. Am J Clin Nutr 2001; 74: 464-73.

[15] Dewailly E, Blanchet C, Gingras LS, Holub BJ. Cardiovascular disease risk factors and n-3 fatty acid status in the adult population of James Bay Cree. Am J Clin Nutr 2002; 76: 85-92.

[16] Dewailly EE, Blanchet C, Gingras S, et al. Relations between n-3 fatty acid status and cardiovascular disease risk factors among Quebecers. Am J Clin Nutr 2001; 74: 603-11.

[17] Gudbjarnason S. Dynamics of n-3 and n-6 fatty acids in phospholipids of heart muscle. J Intern Med Suppl 1989; 731: 117-28.

[18] Cazzola R, Rondanelli M, Russo-Volpe S, Ferrari E, Cestaio B. Decreased membrane fluidity and altered susceptibility to peroxidation and lipid composition in overweight and obese female erythrocytes. J Lipid Res 2004; 45: 1846-51.

[19] Bakewell L, Burdge GC, Calder PC. Polyunsaturated fatty acid concentrations in young men and women consuming their habitual diets. Br J Nutr 2006; 96: 93-9.

[20] Burdge GC, Calder PC. Conversion of alpha-linolenic acid to longer-chain polyunsaturated fatty acids in human adults. Reprod Nutr Dev 2005; 45: 581-97.

[21] Giltay EJ, Gooren LJ, Toorians AW, Katan MB, Zock PL. Docosahexaenoic acid concentrations are higher in women than in men because of estrogenic effects. Am J Clin Nut 2004; 80: 1167-74.

[22] Agostoni C, Marangoni F, Grandi F, et al. Earlier smoking habits are associated with higher serum lipids and lower milk fat and polyunsaturated fatty acid content in the first 6 months of lactation. Eur J Clin Nutr 2003; 57: 1466-72. 
[23] Rise P, Marangoni F, Galli C. Regulation of PUFA metabolism: pharmacological and toxicological aspects. Prostaglandins Leukot Essent Fatty Acids 2002; 67: 85-9.

[24] Leeson CP, Mann A, Kattenhorn M, Deanfield JE, Lucas A, Muller DP. Relationship between circulating n-3 fatty acid concentrations and endothelial function in early adulthood. Eur Heart J 2002; 23: 216-22.

[25] Leng GC, Smith FB, Fowkes FG, et al. Relationship between plasma essential fatty acids and smoking, serum lipids, blood pressure and haemostatic and rheological factors. Prostaglandins Leukot Essent Fatty Acids 1994; 51: 101-8.

[26] Hibbeln JR, Makino KK, Martin CE, Dickerson F, Boronow J, Fenton WS. Smoking, gender, and dietary influences on erythrocyte essential fatty acid composition among patients with schizophrenia or schizoaffective disorder. Biol Psychiatry 2003; 53: 43141.

[27] Simon JA, Fong J, Bernert Jr JT, Browner WS. Relation of smoking and alcohol consumption to serum fatty acids. Am J Epidemiol 1996; 144: 325-34.

[28] Polidori MC, Mecocci P, Stahl W, Sies H. Cigarette smoking cessation increases plasma levels of several antioxidant micronutrients and improves resistance towards oxidative challenge. $\mathrm{Br} \mathrm{J}$ Nutr 2003; 90: 147-50.

[29] Das UN. Essential fatty acid metabolism in patients with essential hypertension, diabetes mellitus and coronary heart disease. Prostaglandins Leukot Essent Fatty Acids 1995; 52: 387-91.

[30] Seigneur M, Freyburger G, Gin H, et al. Serum fatty acid profiles in type I and type II diabetes: metabolic alterations of fatty acids of the main serum lipids. Diabetes Res Clin Pract 1994; 23: 169-77.
[31] Pelikanova T, Kohout M, Valek J, Base J, Stefka Z. Fatty acid composition of serum lipids and erythrocyte membranes in type 2 (non-insulin-dependent) diabetic men. Metabolism 1991; 40: 17580.

[32] Clore JN, Allred J, White D, Li J, Stillman J. The role of plasma fatty acid composition in endogenous glucose production in patients with type 2 diabetes mellitus. Metabolism 2002; 51: 1471-7.

[33] Wang Y, Botolin D, Xu J, et al. Regulation of hepatic fatty acid elongase and desaturase expression in diabetes and obesity. J Lipid Res 2006; 47: 2028-41.

[34] Brenner RR. Hormonal modulation of delta6 and delta5 desaturases: case of diabetes. Prostaglandins Leukot Essent Fatty Acids 2003; 68: 151-62.

[35] Wang C, Harris WS, Chung M, et al. n-3 Fatty acids from fish or fish-oil supplements, but not alpha-linolenic acid, benefit cardiovascular disease outcomes in primary- and secondary-prevention studies: a systematic review. Am J Clin Nutr 2006; 84: 5-17.

[36] Nordestgaard BG, Benn M, Schnohr P, Tybjaerg-Hansen A. Nonfasting triglycerides and risk of myocardial infarction, ischemic heart disease, and death in men and women. JAMA 2007; 298: 299-308.

[37] Lemaitre RN, King IB, Mozaffarian D, et al. Plasma phospholipid trans fatty acids, fatal ischemic heart disease, and sudden cardiac death in older adults: The Cardiovascular Health Study. Circulation 2006; 114: 209-15.

[38] Siscovick DS, Raghunathan TE, King I, et al. Dietary intake and cell membrane levels of long-chain n-3 polyunsaturated fatty acids and the risk of primary cardiac arrest. JAMA 1995; 275: 1363-7.

(C) Block et al.; Licensee Bentham Open.

This is an open access article licensed under the terms of the Creative Commons Attribution Non-Commercial License (http://creativecommons.org/licenses/by-nc/3.0/) which permits unrestricted, non-commercial use, distribution and reproduction in any medium, provided the work is properly cited. 